\title{
Discours
}

Revue de linguistique, psycholinguistique et

informatique. A journal of linguistics, psycholinguistics and computational linguistics

$7 \mid 2010$

Varia

\section{De l'expression du point de vue à l'anticipation textuelle : le rôle de à première vue}

\section{Véronique Lenepveu}

\section{OpenEdition}

Journals

Édition électronique

URL : http://journals.openedition.org/discours/8115

DOI : $10.4000 /$ discours.8115

ISSN : 1963-1723

Éditeur :

Laboratoire LATTICE, Presses universitaires de Caen

\section{Référence électronique}

Véronique Lenepveu, « De l'expression du point de vue à l'anticipation textuelle : le rôle de à première vue », Discours [En ligne], 7 | 2010, mis en ligne le 20 décembre 2010, consulté le 21 décembre 2020 URL : http://journals.openedition.org/discours/8115; DOI : https://doi.org/10.4000/discours.8115

\section{(c) (1) () $\Theta$}

Discours est mis à disposition selon les termes de la licence Creative Commons Attribution - Pas d'Utilisation Commerciale - Pas de Modification 4.0 International. 

Revue de linguistique, psycholinguistique et informatique

\title{
De l'expression du point de vue
}

\section{à l'anticipation textuelle : le rôle de à première vue}

\author{
Véronique Lenepveu \\ Université de Caen Basse-Normandie, CRISCO EA 4255 \\ Campus 1, bâtiment Lettres \\ Esplanade de la Paix \\ 14032 Caen Cedex \\ veronique.lenepveu@unicaen.fr
}

Véronique Lenepveu, «De l'expression du point de vue à l'anticipation textuelle: le rôle de à première vue», Discours, 7 | 2010, [En ligne], mis en ligne le 20 décembre 2010. 



\title{
De l'expression du point de vue à l'anticipation textuelle : le rôle de à première vue
}

\author{
Véronique Lenepveu \\ Université de Caen Basse-Normandie, CRISCO EA 4255
}

\begin{abstract}
Nous nous proposons d'étudier le fonctionnement sémantique et pragmatique de la locution adverbiale à première vue quand elle introduit un point de vue au sens de Nølke (1994a), et qu'elle joue un rôle de «cadratif» prospectif (cf. Charolles, 1997). Dans cette perspective, nous nous sommes attachée à l'étude d'enchaînements discursifs dans lesquels à première vue, en position détachée, fonctionne en corrélation avec des expressions signalant une évolution dans le temps du jugement du locuteur (à mieux regarder, à y regarder de plus près, à bien y réfléchir, en y réfléchissant, tout compte fait, tout bien considéré...). L'étude de ces enchaînements, qui vont parfois au-delà du paragraphe, fait apparaître la capacité de la locution à première vue à initier un cadre et à appeler un autre cadre dans lequel le jugement va être réévalué. En présentant le point de vue qu'elle introduit comme provisoire et en attente de confirmation, la locution anticipe sur la suite du texte et s'inscrit dans une structure aspectuelle de dicto qui est celle d'un procès énonciatif de prise en considération d'une situation en vue de constituer un jugement plus ou moins stabilisé.
\end{abstract}

Mots clés: marqueur de point de vue, cadre, procès énonciatif, aspect de dicto, phases, jugement provisoire/stabilisé

We propose a semantic and pragmatic analysis of the french locution à première vue (lit "at first sight") when it indicates a point of view (in the sense of Nølke, 1994) and plays a role in textual structuration (Charolles, 1997). In this perspective, we study discursive patterns in which à première vue is employed in contrast with expressions which signal an evolution of the judgment of the speaker (à mieux regarder, à y regarder de plus près, à bien y réfléchir, en y réfléchissant, tout compte fait, tout bien considéré...). All these expressions can appear in peripheric positions. They express a de dicto aspectual value, by selecting a phase of an enonciative process which consists in taking in consideration a situation in order to formulate a more or less settled judgment.

Keywords: marker of point of view, frame, enonciative process, de dicto aspect, phases, more or less settled judgment

Langue étudiée: français

Language: French

Marqueurs linguistiques: à première vue, à mieux regarder, tout bien considéré

\section{Introduction}

Nous nous sommes proposé d'étudier le fonctionnement sémantico-pragmatique de la locution adverbiale à première vue quand elle joue le rôle de marqueur de point de vue au sens de Nølke (I994a). Un point de vue comprend un contenu propositionnel et un jugement portant sur ce contenu, jugement lui-même attribué 
à un être discursif distinct du locuteur responsable de l'acte d'énonciation ${ }^{1}$. Dans cette perspective, nous nous sommes attachée en particulier à l'étude d'enchâ̂nements discursifs plus ou moins stéréotypés, qu'on peut qualifier de «routines argumentatives» (Combettes et Kuyumcuyan, 2007), dans lesquels s'inscrit $\grave{a}$ première vue. En effet, très souvent, à première vue entre dans un contraste binaire et fonctionne en corrélation avec une expression signalant un point de vue venant disqualifier sur le plan argumentatif le point de vue introduit précédemment avec $\grave{a}$ première vue. Ainsi, à première vue peut entrer en relation avec un marqueur oppositif (mais, en réalité, en fait...), voire concessif (cependant, pourtant...), ou encore avec une expression nominale qui signale une erreur de jugement (mauvais calcul, erreur, résultat en trompe-l'œil...), éventuellement combinée avec un oppositif ou avec un concessif ${ }^{2}$ :

[I] À première vue, la cour n'a rien d'extraordinaire, à l'exception de sa beauté simple et nue. Pourtant, cette cour est une cage, cette maison est une prison: face à la porte d'entrée, une plaque de protection en métal rouge cache la vue.

(Le Monde, I7/05/oI)

[2] À première vue, le chorégraphe berlinois Félix Ruckert a tout d'un artiste propre sur lui, lesté d'un curriculum vitae impeccable. En réalité, cet ex-interprète de Pina Bausch (de 1992 à 1994) invente depuis sept ans des dispositifs interactifs subtilement perturbants.

(Le Monde, $2 \mathrm{I} / \mathrm{II} / \mathrm{OI}$ )

[3] À première vue, on se disait que jamais on ne se dépêtrerait de tant de monde et de tant d'objets. Erreur cependant; après une heure de route, tout était merveilleusement tassé et immobile.

('Frantext intégral', P. Loti, Le roman d'un spabi, 188I)

À première vue peut également entrer en relation avec des constructions détachées (locutions adverbiales, formes infinitives ou gérondives...) qui apparaissent souvent en position frontale et qui signalent une évolution dans le temps du jugement du locuteur concernant une situation, telles que à mieux regarder, en réfléchissant, à examiner les choses de plus près, ou bien tout compte fait, tout bien considéré, réflexion faite... Ces expressions peuvent, elles aussi, se combiner avec un marqueur d'opposition ou de concession:

1. La voix collective ON, l'allocutaire TU, une tierce personne IL, mais aussi le locuteur L peuvent avoir ce statut d'être discursif, d'où le statut particulier du locuteur qui est à la fois responsable de l'énonciation et source éventuelle de points de vue. Pour des développements ultérieurs de l'analyse de Nølke (I994a) dans le cadre théorique de la ScaPoline (théorie scandinave de la polyphonie linguistique), voir entre autres, Nølke et al. (2004), et plus récemment Birkelund et al. (2009).

2. Le gras et les italiques dans les exemples sont de nous. 
[4] À première vue, on croirait qu'il est inhabité, ce pays; à mieux regarder, cependant, on s'aperçoit combien son opulent manteau vert est déjà sournoisement travaillé en dessous par le microbe humain [...].

('Frantext intégral', P. Loti, Un pellerin d'Angkor, 19ı2)

[5] Quel âge peut-il avoir? À première vue une douzaine d'années. À y regarder mieux, davantage sûrement - quinze ans peut-être, car il paraissait naturellement fluet.

('Frantext intégral', M. Tournier, Les météores, 1975)

Nous nous intéressons précisément à ce dernier type d'enchaînements pour montrer comment à première vue est à même de jouer un rôle d'organisateur textuel, c'est-à-dire, dans les termes de Charolles (1997), de «cadratif» prospectif, et, au bout du compte, comment l'analyse d'enchaînements discursifs, qui vont souvent au-delà du paragraphe, permet de comprendre le fonctionnement de ce type de locution adverbiale. Dans cette perspective, après avoir identifié les conditions d'emploi de la locution adverbiale à première vue quand elle s'interprète comme marqueur de point de vue, lequel se donne comme provisoire, nous montrerons que l'évolution du point de vue du locuteur peut se décrire en termes de structure phasale d'un même procès énonciatif de prise en considération d'une situation en vue de constituer un jugement stabilisé, et que les expressions du type à première vue, à bien y regarder, tout bien considéré... servent à sélectionner différentes phases de ce procès énonciatif. Les exemples sont choisis dans la base de données 'Frantext intégral' ainsi que dans Le Monde sur CD-ROM (1995-1996, 1999-2002).

\section{Les deux emplois de à première vue}

Dans le Trésor de la langue française (1994, t. XVI : 1373), les expressions à première vue et à la première vue sont citées, à l'entrée vue, parmi «les locutions évoquant l'usage de ce sens pour évaluer, faire quelque chose». Ces deux expressions peuvent signifier «au premier coup d'œil». Parmi les exemples proposés, nous citons:

[6] Gérard lui avait été sympathique à première vue.

(É. Peisson, Parti de Liverpool..., Paris, Grasset, 1932, p. 120)

[7] Werner croit [...] que les êtres créés l'un pour l'autre doivent se reconnoître à la première vue.

( $\mathrm{M}^{\text {me }}$ de Staël, De l'Allemagne, Paris, H. Nicolle, i8ro, t. 3, p. 139)

Mais il est précisé également que, prise dans un sens figuré, la locution à première vue est paraphrasable par «sans examiner les choses de près, sans réfléchir»:

[8] Un peu de travail de bureau, alternant avec de longues sorties [...] hein? À première vue, qu'est-ce que vous en diriez?

(J. Romains, Les hommes de bonne volonté, Paris, Flammarion, 1932, p. 24I) 
Nous verrons que à première vue peut effectivement garder une signification relativement compositionnelle comme à la première vue, mais peut aussi, par extension, introduire un point de vue susceptible d'être confirmé ou infirmé par la suite du texte. Les deux valeurs sémantiques sont corrélées au fonctionnement syntaxique de la locution, ce qu'il est possible de décrire à partir de la double opposition que propose Guimier (1996) entre adverbes intra-prédicatifs/extra-prédicatifs et entre adverbes endophrastiques/exophrastiques. Une étude très rapide de l'évolution diachronique des locutions à la première vue et à première vue au moyen de la base de données 'Frantext intégral' permet de montrer dans quelle mesure le figement de la locution au niveau morphologique est associé à des modifications dans son fonctionnement syntaxique et sémantique.

\subsection{De à la première vue vers à première vue}

La première occurrence de à la première vue repérable dans 'Frantext intégral' date de $1637^{3}$ et la dernière de $193 \mathrm{I}$; la locution signifie «la première fois que $\mathrm{X}$ a l'expérience de $\mathrm{Y}$ », où $\mathrm{X}$ et $\mathrm{Y}$ s'instancient en fonction du contexte et où l'expérience est le plus souvent, mais non nécessairement, visuelle ${ }^{4}$. Elle se paraphrase par «au premier coup d'œil» ou «la première fois que $\mathrm{X}$ voit $\mathrm{Y} »$ :

[9] [...] il jura qu'il me passerait son épée au travers du corps et m’exterminerait à la première vue.

('Frantext intégral', A.-R. Lesage, Histoire de Gil Blas de Santillane, 1732)

[io] [...] cher M. Reves, les honnêtes gens doivent s'aimer à la première vue.

('Frantext intégral', l'abbé Prévost, Nouvelles lettres angloises ou bistoire du chevalier Grandisson, 1755)

[II] D'ailleurs, je lui devais la vérité, puisqu'il me donnait sa confiance quasiment à la première vue.

('Frantext intégral', G. Sand, Les maitres sonneurs, I86o, Dixième veillée)

Dans la perspective de Guimier (1996) ${ }^{5}$, la locution présente ici, sur le plan sémantique, un fonctionnement endophrastique dans la mesure où elle caractérise

3. B. Fagard me fait cependant remarquer qu'on trouve des occurrences de constructions similaires avant cette date: «à cette premiere veue» (I6IO), «à cestre premiere veue» (I6I2), «dés la premiere veue» (I6IO), «à nostre premiere veue» (I6I3), «à sa premiere veue» (I637), «à une première vue» (I69I). On trouve aussi l'expression nominale «la premiere veue» (I6IO). Les datations à partir de Frantext ne peuvent évidemment être qu'indicatives quant à la période d'apparition de ces locutions dans la langue.

4. À première vue n'implique pas systématiquement un rapport visuel, comme l'atteste l'exemple [i] qui suit: [i] Quels sont les noms qui frappent à la première vue jetée sur l'histoire de cette époque? ('Frantext intégral', E. Renan, L'avenir de la science, I89o).

5. L'opposition endophrastique/exophrastique est une opposition sémantique; nous citons Guimier (I996: 6): «[Les adverbes endophrastiques] sont, d’un point de vue sémantique, des constituants internes à la phrase, qui affectent le contenu même de l'élément sur lequel ils portent et, ce faisant, participent à la construction du sens référentiel de la phrase [...]. Les adverbes exophrastiques sont des constituants externes à la phrase, en ce sens qu'ils ne participent pas à la construction de son sens référentiel, mais 
une circonstance du procès, ce qu'attestent les paraphrases proposées, et sur le plan syntaxique, un fonctionnement intra-prédicatif puisqu'elle est intégrée à la phrase et dépendante d'un constituant de la phrase; en l'occurrence, dans les exemples choisis, elle est dépendante du verbe, et peut se placer après celui-ci sans rupture intonative. Mais elle peut apparaitre également en position détachée, préverbale ou postverbale, c'est-à-dire en emploi extra-prédicatif (tout en restant endophrastique, sur le plan sémantique):

[I2] A la première vue de monsieur, l'amour fit évanouir les espérances de fortune que j'avois fondées sur mes attraits.

('Frantext intégral', comte de Caylus, Histoire de Monsieur Guillaume, cocher, 1737)

[13] Je lui dirai, à la première vue, que je vous ai montré sa lettre, que vous l'avez regardée d'un air glacé, et qu'enfin, sans la lire, vous l'avez déchirée avec un mépris froid [...]. ('Frantext intégral', A.-R. Lesage, Histoire de Gil Blas de Santillane, 1732)

[I4] Ses mains étaient jointes, ses yeux étaient fixes. À la première vue on la prenait pour un spectre, à la seconde pour une statue.

('Frantext intégral', V. Hugo, Notre-Dame de Paris, I832)

\subsection{Un emploi endophrastique intra- ou extra-prédicatif de à première vue}

Si on consulte toujours 'Frantext intégral', les premières occurrences de à première $v u e^{6}$ apparaissent au début du XIX ${ }^{e}$ siècle. La modification morphologique peut être interprétée comme l'indice d'un plus grand figement syntaxique puisque à première vue n'accepte pas de complément contrairement à la locution à la première vue qui, au vu de l'exemple [12], ne semble pas totalement figée. Sur le plan sémantique, ’̀ première vue participe, dans ces exemples, à la construction du sens référentiel de la phrase en caractérisant le procès: la locution est donc endophrastique comme à la première vue. Elle peut se paraphraser par «au premier coup d'œil» ou «la première fois que $\mathrm{X}$ voit $\mathrm{Y}$ ", ou encore au moyen des adverbes d'aspect aussitôt et immédiatement qui marquent le mode de survenance du procès, à première vue n'impliquant pas systématiquement un rapport visuel comme l'atteste l'exemple [17] ci-dessous. Sur le plan syntaxique, elle peut occuper la position canonique postverbale de l'adverbe intra-prédicatif comme ci-dessous:

[ı] On me présenta à lui, comme une petite merveille, et lorsque j'eus déchiffré facilement un morceau à première vue, il me prit en amitié [...].

('Frantext intégral', G. Sand, Consuelo, I843)

[16] Depuis ce jour, je n'ai plus su mépriser une femme à première vue.

('Frantext intégral', A. Dumas fils, La dame aux camélias, 1848)

représentent des traces de l'intervention du locuteur, qui commente tout ou partie de son énoncé ou de l'acte qui le produit».

6. La première occurrence repérée dans 'Frantext intégral' date de I828. 
[I7] Voulez-vous installer à la tête de ce comité Bossuet, l'écrivain le plus fait assurément pour entendre à première vue la grande façon de Pascal, ainsi surprise?

('Frantext intégral', C.-A. Sainte-Beuve, Port-Royal, I848)

Cet emploi se maintient au $\mathrm{XX}^{\mathrm{e}}$ siècle ${ }^{7}$ :

[18] Les indications qui suivent sont destinées à faciliter l'identification des étoiles. Elles se distinguent à première vue par leur éclat.

('Frantext intégral', A. Danjon, Cosmographie: classe de mathématiques, 1948)

[19] La nécessité d'établir un catalogue d'étoiles a surgi tout naturellement du désir de reconnaître à première vue la présence d'une comète ou d'une nova dans le ciel.

('Frantext intégral', E. Schatzman, Astrophysique, 1963)

On observe que la locution est à même de constituer le focus de la négation ou de l'interrogation, indices de son intégration à la phrase:

[20] Je vois très vite et je juge assez bien. Et vous? Vous faites-vous à première vue une idée des gens?

('Frantext intégral', G. Sand, Le marquis de Villemer, i864)

[2I] Oh! si, si, comme votre nez s'est allongé, comme il a gonflé... je ne m’en étais pas aperçu à première vue, mais je m’en aperçois... terriblement allongé... Ah! quel dommage!

('Frantext intégral', E. Ionesco, Les chaises, 1954)

Et il faut préciser que, là encore, la locution peut rester dans la portée du verbe sur le plan sémantique, et apparaître en position détachée, c'est-à-dire en position extra-prédicative du point de vue syntaxique:

[22] - j'aime ou j'abhorre, dans l'acception physique du mot; à première vue, en un clin d'œil, j'ai jugé un homme ou une femme pour jamais.

('Frantext intégral', A. de Lamartine, Souvenirs, impressions, pensées et paysages pendant un voyage en Orient (I832-I833) ou notes d'un voyageur, I835)

[23] À première vue, Drifter le jaugea comme un décorateur de cinéma ou un «créatif» de publicité.

('Frantext intégral', P. Labro, Desbateaux dans la nuit, I982)

\subsection{Un emploi exophrastique extra-prédicatif de à première vue}

Parallèlement, dès le milieu du XIX ${ }^{e}$ siècle, on trouve dans Frantext des exemples où la locution à première vue n'est plus régie par le verbe ou par un autre constituant

7. Outre les exemples choisis dans 'Frantext intégral', on peut citer un exemple [i] pris dans Le Monde: [i] [...] sa capacité à maîtriser à première vue le moindre détail des œuvres qu'il dirigeait n'avait d'égales que sa curiosité et sa hardiesse (Le Monde, I8/03/96). 
de la phrase: elle s'interprète alors comme marqueur de point de vue, dans le sens où elle indique une attitude du locuteur relativement à un jugement portant sur un contenu propositionnel, à savoir que ce jugement a été formulé «sans examiner les choses de près, sans réfléchir». Un exemple attesté en I850:

[24] À première vue, il semble que le grand désavantage du métayage soit pour les propriétaires; à examiner les choses de plus près, on voit que les métayers se trouvent au plus bas degré de la pauvreté et parfois de la misère...

('Frantext intégral', O. Voillard et al. (eds), Documents d'bistoire contemporaine, I850)

Dans cet emploi qui devient majoritaire au $\mathrm{XX}^{\mathrm{e}}$ siècle, à première vue reste hors du champ de la négation et de l'interrogation, ce qui est une caractéristique de l'emploi adverbial extra-prédicatif exophrastique. Exemples:

[25] Entre psychologues, sociologues et historiens, quel serait à première vue l'enjeu d'un débat de compétence et d'attributions?

('Frantext intégral', L. Febvre, Combats pour l'bistoire, 1952)

[26] À première vue, l'arme du crime n'était pas restée sur les lieux [...].

('Frantext intégral', J.-P. Manchette, Morgue pleine, 1973)

Outre ces tests de l'interrogation et de la négation, le critère de la coordination permet de vérifier la coexistence de deux emplois pour à première vue. En emploi intra-prédicatif, la locution, qui se coordonne dans l'exemple [27] avec un complément de lieu, dépend syntaxiquement du verbe. En emploi extra-prédicatif dans les exemples [28] et [29], elle se coordonne avec un élément détaché, marqueur de point de vue:

[27] Le devant du corps était griffé et mordu, pour autant qu'on puisse en juger à première vue et à deux mètres de distance.

('Frantext intégral', J.-P. Manchette, Morgue pleine, 1973)

[28] Expérimentalement et à première vue, l'univers considéré dans son fonctionnement mécanique ne se présente pas à nous comme un quantum ouvert capable d'embrasser dans son angle un réel toujours plus grand [...].

('Frantext intégral', P. Teilhard de Chardin, Le phénomène bumain, 1955)

[29] À première vue et pour un lecteur détaché, ce sont des aventures inquiétantes qui enlèvent des personnages tremblants et entêtés à la poursuite de problèmes [...].

('Frantext intégral', A. Camus, Le mythe de Sisyphe, 1942)

Si on resitue rapidement l'évolution de la locution de à la première vue vers à première vue dans la perspective de Traugott (1995) et Combettes (1995), on peut observer que la modification morphologique de la locution n'entraîne pas forcément de modification quant au niveau d'intégration syntaxique de la locution, puisque $\grave{a}$ première vue peut toujours faire partie du contenu propositionnel, rester attachée au 
syntagme verbal (ou à un autre syntagme) et se paraphraser par «au premier coup d'œil». Mais la locution peut acquérir une certaine autonomie syntaxique, et par là une portée plus large, comme l'attestent les critères de l'interrogation et de la négation. Sa fonction devient modalisatrice dans le sens où elle indique une attitude, une prise de position du locuteur par rapport à son énonciation. À première vue, qui gagne ainsi en «subjectivité» (Traugott, 1995: 45), devient marqueur de point de vue et se paraphrase par «sans examiner les choses de près, sans réfléchir». Ce qui est mis évidence dans la figure I:

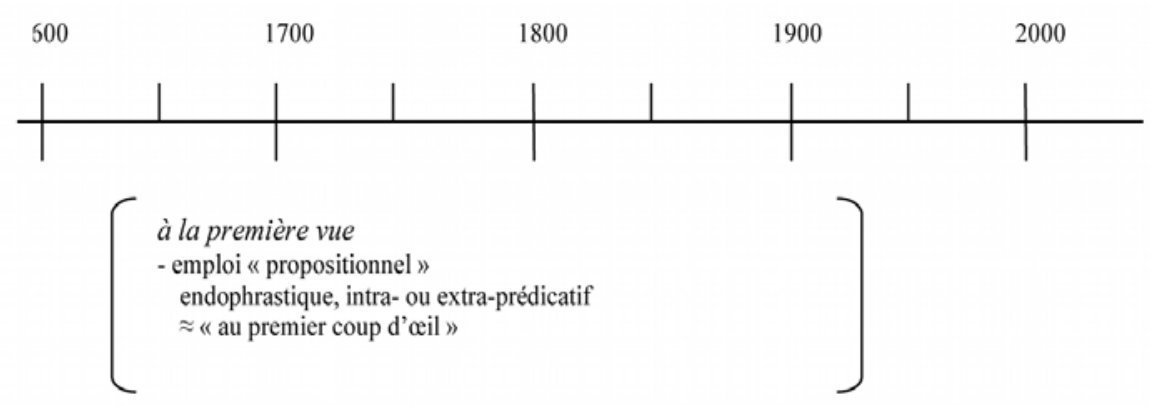

1637

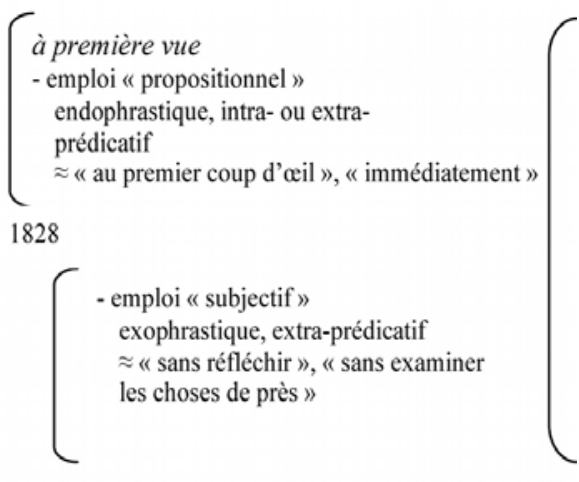

1850

Fig. 1 : évolution diachronique des emplois des locutions à la première vue et à première vue, d'après l'examen des occurrences dans 'Frantext intégral'

Reste que la position initiale détachée, qui garantit le plus souvent le changement de statut des adverbiaux (Combettes, 1995; Combettes et Kuyumcuyan, 2007), ne peut constituer ici un critère distinctif entre les deux emplois «propositionnel» et «subjectif» puisque la locution peut demeurer endophrastique dans cette position. Le cotexte se révèle alors souvent déterminant pour interpréter à première vue comme marqueur de point de vue. Si nous nous limitons au cadre de la phrase, nous observons que la présence d'éléments modalisateurs comme sembler et paraître ainsi que l'emploi du conditionnel favorisent l'interprétation de à première vue comme marqueur de point de vue. Au-delà de la phrase, nous nous attachons à la mise en contraste possible de à première vue avec une expression qui introduit un jugement 
venant disqualifier le point de vue précédemment introduit par cette locution ${ }^{8}$. Précisons que des exemples attestés, dès le $\mathrm{XIX}^{\mathrm{e}}$ siècle, montrent que le point de vue introduit avec à première vue n'est pas systématiquement neutralisé sur le plan argumentatif et qu'il peut rester en attente de confirmation:

[30] Je vous renvoie les articles de Considérant. C'est très remarquable, très intéressant et à première vue très praticable. Au reste, facile ou non à réaliser, il faut dire avec lui, c'est nécessaire.

('Frantext intégral', G. Sand, Correspondance, I85I)

Mais quel que soit le cas de figure envisagé (point de vue resté ou non en attente de confirmation), on peut poser l'hypothèse que la locution, dans son emploi extraprédicatif exophrastique, signale toujours un point de vue donné comme provisoire, et qu'elle appelle une suite, ne serait-ce que virtuelle, comme dans l'exemple ci-dessous:

[3I] La découverte a eu lieu le jeudi 23 août dans la grotte dénommée Caune de l'Arago. Cette mandibule appartient à un anténéandertalien - Homo erectus européen. «Elle n'a pas encore été sortie de terre, car nous voulons la mouler avant de l'enlever, précise Henry de Lumley. Nous n'avons donc pas encore pu l'étudier. Mais, à première vue, elle semble assez gracile, et pourrait appartenir à une femme».

(Le Monde, 27/o8/or)

L'analyse de la cooccurrence de à première vue avec les verbes de perception sembler et paraître ainsi qu'avec le conditionnel nous permettra de préciser l'origine de ce point de vue donné comme provisoire. Nous verrons en effet comment la locution peut contraindre la valeur évidentielle de l'énoncé (Chafe, I986; Dendale et Tasmowski, 1994), même si elle n'indique pas à proprement parler la source du savoir et ne peut être considérée comme un marqueur lexical d'évidentialité ${ }^{9}$. Enfin, l'étude de la locution, dans son emploi contrastif, lorsqu'elle entre en relation avec une expression qui signale explicitement une évolution dans le temps du jugement du locuteur, mettra en évidence à la fois son fonctionnement comme marqueur de point de vue et sa capacité cadrative.

8. Certains environnements, autorisant une double lecture, attestent de l'évolution possible de la locution d'un emploi endophrastique vers un emploi exophrastique. Pour exemple [i] :

[i] Donc, ce temple d'Hathor, construit aux temps de Cléopâtre et d'Auguste, sur un emplacement vénéré de toute antiquité, rappelle à première vue quelque conception des Ramsès. Cependant, si l'on regarde mieux, c'est dans le détail surtout des milliers de figures en bas-relief que l'écart se montre considérable [...] ('Frantext intégral', P. Loti, La mort de Philoe, 1909).

9. Un marqueur dit d'évidentialité, traduit de l'anglais evidentiality par Vet (I988), est défini de la façon suivante chez Dendale et Tasmowski (1994: 5): «Un marqueur évidentiel est une expression langagière qui apparaît dans l'énoncé et qui indique si l'information transmise dans cet énoncé a été empruntée par le locuteur à autrui ou si elle a été créée par le locuteur lui-même, moyennant une inférence ou une perception». Voir aussi dans cette perspective sémantique et conceptuelle, les travaux antérieurs de Chafe (I986) pour l'anglais. 


\section{3. À première vue, marqueur de point de vue}

\subsection{Cooccurrence de à première vue avec sembler et paraître}

Si l'on admet que sembler et paraître ont la particularité de présenter un jugement comme résultant d'indices qui ne sont pas suffisants, c'est-à-dire qui ne peuvent avoir valeur de tekmerion ${ }^{10}$ au sens aristotélicien du terme (Rhétorique I, 2, I357 b 3-10), nous pouvons comprendre la convergence entre les instructions associées à sembler et paraître et celles qui sont associées à la locution à première vue. Avec à première vue, le jugement se donne comme provisoire, et ce caractère provisoire s'explique par le caractère non décisif des indices sur lesquels se fonde le jugement. Les deux marques sont ainsi convergentes en ce qu'elles contribuent toutes deux à l'effet de distance du locuteur relativement au jugement porté. Des exemples:

[32] À première vue, l'affaire paraît claire, un crime crapuleux quelconque [...].

('Frantext intégral', G. Bernanos, Un crime crapuleux, 1935)

[33] Le plan semble à première vue compliqué à cause du nombre des éléments, mais il se révèle souple et bien étudié.

('Frantext intégral', J. Cain (dir.), La civilisation écrite, 1939)

La comparaison que propose Nølke (1994b) des tours impersonnels il semble que $p$ et il paraît que $p$ dans le cadre d'une analyse polyphonique, est fondée en partie sur une étude de Bourdin (1988) et sur la distinction faite par Ducrot (1984) entre le locuteur «en tant que tel», responsable de l'énonciation, et le locuteur «en tant qu'être du monde», existant indépendamment de l'acte d'énonciation (locuteur-entant-qu'individu chez Nølke); elle nous permettra de comprendre le fonctionnement de à première vue. Les deux tournures, selon Nølke (I994b), marquent une dilution linguistique des responsabilités et indiquent que la responsabilité du point de vue véhiculé par $p$ est attribuée à un être discursif distinct du locuteur «en tant que tel» (locuteur-en-tant-que-tel), mais il semble que et il paraît que induisent des structures polyphoniques différentes. Dans son emploi le plus courant, il paraît que signale une source extérieure de type ouï-dire et attribue le point de vue véhiculé par $p$ à une instance distincte du locuteur-en-tant-qu'individu. En revanche, il semble que introduit une inférence à partir d'un certain nombre de signes ou d'indices plus ou moins inconscients, inférence donnée comme le résultat d'un dialogue intériorisé ayant lieu au moment de l'énonciation et qui, en conséquence, ne peut être mise en rapport avec une source extérieure de type ouï-dire. L'être discursif responsable du point de vue est alors identifié au locuteur-en-tant-qu'individu, ce qui peut être explicité au moyen du datif me (il me semble que p). Or, il se trouve que la locution à première vue est parfaitement compatible avec il semble que:

10. Le tekmerion est un indice qui conduit nécessairement à une seule et unique conclusion (par exemple, la fièvre pour la maladie). Sur les indices, voir aussi Anscombre et al. (2009). 
[34] À première vue, il semble que cette réponse s'accorde assez bien avec notre thèse mécaniste, mais ce n'est qu'une apparence [...].

('Frantext intégral', R. Ruyer, Esquisse d'une philosophie de la structure, 1930)

En revanche, elle ne se combine pas avec il paraît que, ni avec aucun autre marqueur de source externe au locuteur $\left(?^{*} \grave{A}\right.$ première vue, il paraît que Marie est malade / $*^{*} \grave{A}$ première vue, on dit que Marie est malade), d'où notre hypothèse selon laquelle à première vue exclut un jugement fondé sur le discours d'autrui. L'examen des contraintes qui pèsent sur la combinaison de la locution avec un conditionnel confirme notre analyse. Là encore, il y a convergence entre les instructions associées au conditionnel et les instructions associées à la locution, dans la mesure où à première vue autorise et contribue à l'effet de mise à distance de l'assertion marqué par ce conditionnel, mais la locution exclut toujours que la responsabilité du propos soit attribuée à une tierce personne.

\subsection{Cooccurrence de à première vue avec le conditionnel}

Combiné à la locution à première vue, le fonctionnement du conditionnel ne peut s'apparenter au conditionnel dit d'emprunt, illustré le plus souvent par le conditionnel journalistique (Abouda, 200I; Gosselin, 200I; Haillet, 2002). Ainsi, dans l'exemple [35] ci-dessous, nous ne pouvons pas utiliser la glose en paraît-il qui permet chez Haillet (2002) d'identifier le conditionnel journalistique comme conditionnel d'altérité énonciative :

[35] À première vue, on pourrait penser que le réchauffement devrait bénéficier à cet oiseau atypique qui réussit le tour de force de se reproduire durant le terrible hiver de l'Antarctique $[. ..] / * \grave{A}$ première vue, on peut penser, paraît-il, que le réchauffement... (Le Monde, $\mathrm{i2} / 05 / 0 \mathrm{I})$

ce qui montre encore une fois que à première vue n'est pas compatible avec une source extérieure du type ouï-dire. Les croyances ou pensées peuvent être explicitement attribuées à une voix collective (on pourrait penser que p), mais qui n'exclut jamais le locuteur. L'être discursif responsable du point de vue évoqué peut être identifié à tout individu ayant l'expérience de l'état de choses en question au moment de l'énonciation, y compris le locuteur en tant qu'individu existant indépendamment de l'acte d'énonciation. Le conditionnel est, dans le contexte donné, un conditionnel hypothétique, le cadre hypothétique étant mis en place au moyen de la locution à première vue qui exprime une condition sous laquelle une croyance ou une pensée sont envisagées et qui se paraphrase de la façon suivante: si on considère la situation à première vue. La proposition n'est validée que dans l'hypothèse avancée, d'où l'effet de sens d'atténuation associé au conditionnel, d'où également la coordination possible de à première vue avec une subordonnée hypothétique:

[36] Dans cette phénoménologie, où à première vue et si on se laisse inspirer par des schémas traditionnels, les motifs de conflits ou de tensions paraissent nombreux [...]. ('Frantext intégral', J. Derrida, L'écriture et la différence, 1967) 
Cette interprétation du conditionnel est favorisée par la présence de verbes d'attitude propositionnelle, modalisateurs d'assertion tels les verbes croire, penser ou encore dire ${ }^{11}$ qui, au conditionnel, ne joue plus le rôle de verbe illocutoire; elle l'est aussi par la présence du modal pouvoir ${ }^{12}$ :

[37] À première vue, on croirait que la parole entendue ne peut rien lui apporter: c'est lui qui donne leur sens aux mots, aux phrases [...].

('Frantext intégral', M. Merleau-Ponty, Phénoménologie de la perception, 1945)

[38] À première vue, on dirait que l'arbitre est fait pour l'économie contemporaine, qui ne connaît ni État ni frontières.

(Le Monde, $\mathrm{I} 8 / 05 / \mathrm{or}$ )

[39] On pourrait croire, à première vue, qu'elles auront le même caractère que celles d'autrefois. Cependant, l'Assemblée qui en sortira ne se trouvera pas du tout dans les mêmes conditions [...].

('Frantext intégral', C. de Gaulle, Discours et messages, 3. Avec le renouveau, 1958-1962, 1970)

[40] Les visites d'usines, dont on penserait à première vue qu'elles dérangent les ouvriers, les distrayant et les écartant de la concentration sur leur tâche, ont pour effet tout au contraire, d'augmenter le rendement [...].

('Frantext intégral', G. Friedmann, Où va le travail humain?, 1950)

Le conditionnel hypothétique tend ici à modaliser l'énonciation en présence d'unités lexicales qui, combinées à l'emploi du conditionnel et à la locution à première vue, visent elles-mêmes à réduire la responsabilité énonciative du locuteur ${ }^{13}$, favorisant ainsi la mise à distance de l'assertion et de ce fait l'emploi contrastif de la locution à première vue.

Dans la classification des adverbiaux de Molinier et Levrier (2000), nous pourrions apparenter à première vue aux «disjonctifs de style» se rapportant à la source

11. Suivant les analyses de Myong Soon (2004), les deux marqueurs on dit que $p$ et on dirait que $p$ indiquent une dilution linguistique des responsabilités, mais diffèrent sur le plan évidentiel: on dit que $p$ marque le ouï-dire alors qu'avec on dirait que $p$, le locuteur partage le point de vue de $p$, lequel est fondé sur une information obtenue par inférence à partir d'indices, tout en manifestant une certaine réserve.

12. Si nous admettons que le rôle de pouvoir est de poser que «toutes les conditions nécessaires à l'actualisation du procès sont réunies au moment considéré», autrement dit, que «rien ne s'oppose à la réalisation du procès» (Fuchs, 1989: 5) et que les chances de réalisation varient selon les contextes, nous pouvons considérer que la présence de la locution et l'emploi du conditionnel contribuent à maintenir la virtualité maximale du procès et favorisent ainsi l'enchaînement avec un discours contradictoire.

13. Korzen et Nølke (I990; 200I) ont montré que des unités lexicales appréciatives, susceptibles de jouer un rôle de modificateur «déréalisant atténuateur» (Ducrot, 1995; Lenepveu, 2007a), favorisent une lecture du conditionnel hypothétique comme modalisateur de l'énonciation. Ainsi, dans l'exemple [i] qui suit, où être tenté de atténue la force argumentative de l'énoncé sans inverser son orientation, le conditionnel hypothétique tend à modaliser l'énonciation et à réduire la responsabilité énonciative du locuteur: [i] [...] à première vue, on serait tenté de regarder la brachycéphalie comme due à l'altitude de ces aires respectives ('Frantext intégral', A.-C. Haddon, Les races bumaines, 1930). 
de l'énoncé, et plus précisément aux formes qui «expriment une réserve prudente du locuteur par rapport à l'information qu'il transmet» (Molinier et Levrier, 2000: 70) et qui en limitent ainsi le crédit auprès du destinataire ${ }^{14}$. Avec à première vue, le locuteur relativiserait la portée de son propos comme il relativise ses propos au moyen d'expressions du type à ma connaissance ou encore d'après ce que je sais, à ce que je crois $^{15}$, à ceci près que la locution ne comprend aucun élément déictique de la première personne et que le locuteur peut s'inscrire explicitement dans une voix collective ( $\bar{A}$ première vue, on se disait que $p$ ). En revanche, au sein des disjonctifs, on isolera d'emblée la locution à première vue des adverbiaux d'emprunt à une source extérieure (à ce qu'il paraît, d'après N...), qui sont typiquement évidentiels (Chafe, 1986; Dendale et Tasmowski, 1994) ou médiatifs ${ }^{16}$ (Guentchéva (ed.), 1996) en ce sens qu'ils explicitent la source du savoir, et par là impliquent une certaine distance prise par le locuteur relativement au jugement porté. À première vue n'explicite pas à proprement parler la source de l'information puisque la locution n'indique pas comment le locuteur a eu accès à l'information qu'il transmet dans son énoncé. Précisément, si nous admettons les trois types de source du savoir généralement admis - perception, inférence et ouï-dire -, la locution ne nous dit pas si le jugement est fondé sur le témoignage des sens, c'est-à-dire sur la perception, ou s'il est obtenu par inférence, mais elle exclut que le jugement soit fondé sur un savoir emprunté.

C'est la possibilité de mise en contraste, due manifestement à la valeur paradigmatisante ${ }^{17}$ de l'ordinal premier (Schnedecker, 2002), que nous voulons maintenant étudier.

\section{4. À première vue, marqueur textuel d'anticipation}

\section{1. À première vue/à mieux y regarder/en y regardant de plus près}

Lorsque la locution met en concurrence deux points de vue s'opposant sur le plan argumentatif, à première vue assure une fonction textuelle dans le sens où elle initie un cadre et appelle un autre cadre dans la suite du texte, cadre dans lequel le jugement va être réévalué. Cette capacité à annoncer un autre cadre vient, nous l'avons vu, de ce

14. Dans la classification plus récente de Molinier (2009), à première vue ferait partie des adverbes distanciatifs (pour autant que je sache, à ma connaissance, si je ne me trompe pas...), sous-groupe d'adverbes énonciatifs qui s'oppose aux adverbes de conviction personnelle (à mon avis, pour moi, d'après moi, selon moi...), aux adverbes d'emprunt à une source extérieure (à ce qu'il paraît, d'après $N$, à ce que j'ai entendu dire...) et aux adverbes d'individuation (personnellement, pour ma part, en ce qui me concerne...).

15. Pour ces expressions, voir Borillo (2004).

16. Nous prenons ici évidentialité et médiation comme synonymes à l’instar de Kronning (2004: I6 sq.). Le terme médiatif est introduit par Lazard (1956) et repris notamment par Guentchéva (1996).

17. À première vue peut d'ailleurs entrer en corrélation avec à seconde vue ou à la seconde vue même si les exemples sont rares:

[i] - Une enveloppe, à première vue anodine. À seconde vue: de la dynamite! ('Frantext intégral', F. Dorin, Les vendanges tardives, 1997). 
qu'à première vue signale toujours un point de vue qui est donné comme provisoire, et qui appelle une suite au moins virtuelle. $\grave{A}$ première vue va ainsi s'opposer sur le plan de la temporalité de dicto à des expressions (locutions adverbiales, formes infinitives, gérondives...) qui apparaissent généralement en position détachée à gauche, et qui concernent aussi la prise en charge des propositions. Dans les exemples que nous avons choisis, la relation que nous établissons entre à première vue et les éléments mis en caractères gras suppose une ordination et un décalage temporel entre ce que le locuteur a pu penser et ce qu'il pense ensuite. Ce décalage temporel est de nature de dicto, la temporalité de dicto étant définie ici, au sens de R. Martin (1987: III-II7), comme celle de la prise en charge de l'énoncé, correspondant à la «fluctuation des univers de croyance». Un premier exemple:

[41] À première vue, l'itinéraire de Yann Gaillard, inspecteur des finances entré par hasard en politique, écrivain subtil qui a consacré six volumes insolites à l'éloge des «morts illustres», peut apparaitre atypique. [...]

Mais à considérer les choses de plus près, l'ancien énarque passé de l'administration aux cabinets ministériels puis à la banque avant de devenir sénateur en 1994, à l'âge de 58 ans, est assez représentatif d'une génération de hauts fonctionnaires à la personnalité affirmée [...].

(Le Monde, 05/05/00)

Pour rendre compte du fonctionnement de ces expressions que nous mettons en relation avec à première vue, nous faisons l'hypothèse d'un procès énonciatif qui consiste à prendre en considération une situation dans son ensemble et qui vise à constituer un jugement stabilisé, et nous posons qu'à l'intérieur de la temporalité de dicto, les éléments que nous mettons en relation servent à sélectionner des phases de ce procès énonciatif. Dans la mesure où ils concernent la structure temporelle interne du procès énonciatif en question, nous dirons que ces éléments prennent une valeur aspectuelle de dicto, chacune des expressions étudiées renvoyant spécifiquement à telle ou telle phase de cette structure aspectuelle. Précisément, nous admettons à la suite de Vet (2002: 182-183, 2008: 458), Tournadre (2004: 23) et Gosselin (2010, 20II) que tout procès est virtuellement décomposable en cinq phases: préparatoire, initiale, médiane, finale et résultante, et nous pensons que de la même façon, le procès énonciatif de prise en considération d'une situation peut se décomposer en cinq phases distinctes que des expressions permettent de signaler (au moins dans certains emplois).

Si nous admettons que le jugement porté par le locuteur sur une situation est présenté avec à première vue comme provisoire parce que le plus souvent spontané, fait dans l'immédiateté et en attente d'une confirmation, nous dirons que à première vue permet de retenir la phase initiale du procès énonciatif de prise en considération d'une situation, lequel peut être pris dans son déroulement avec notamment ${ }^{18}$ les

18. On a également noté que à première vue pouvait entrer en relation, au niveau énonciatif, avec une subordonnée temporelle (quand on y regarde de plus près), une subordonnée hypothétique (si on y regarde de plus près), ou encore une phrase impérative (regardons-y de plus près). 
constructions infinitives en $\grave{a}+$ Vinf comme à y regarder de plus près, à mieux regarder, $\grave{a}$ examiner les choses de plus près, ou bien encore avec les constructions gérondives en regardant bien, en y réfléchissant, en approfondissant... Ces expressions comportent toutes un verbe agentif ${ }^{19}$ qui indique que le sujet devient agent du procès de prise en considération (regarder, considérer, examiner...); en plaçant la prédication principale dans leur portée, elles favorisent, de par leur aspect inaccompli, un mouvement dynamique inaccompli et progressif au niveau de dicto et sélectionnent ainsi la phase médiane du procès énonciatif, lequel consiste à parcourir un ensemble d'éléments relatifs à une situation. Outre l'exemple précédemment cité, on peut proposer les exemples ci-dessous:

[42] Le calendrier politique, surtout, change la donne du tout au tout. À première vue, il apparait rassurant pour la droite. Pour sortir de sa dépression, celle-ci dispose, normalement, de deux années vierges de tout rendez-vous électoral avant les municipales et de trois ans avant la prochaine élection présidentielle. À mieux regarder, cependant, ce délai long est calamiteux, dès lors que l'échéance présidentielle reste le moment clé de la vie politique française, l'occasion décisive où se construisent projets et stratégies.

(Le Monde, 26/o6/99)

[43] En 1994, M. Dominici apprend que ce duo, qu'il qualifie de «faux facturiers professionnels», avait constitué, en mai 1990, en Guadeloupe, la Société caraïbe d'études (SCE). À première vue, rien d'étonnant: à l'époque, M. Boucheron était toujours en place, et les activités de M. Gabaude le conduisaient périodiquement dans les paradis fiscaux des Caraïbes, notamment à Grand-Caïman. En y regardant de plus près, l'ancien chômeur découvre que la SCE est domiciliée à Gourbeyre, qui était alors le fief municipal de Mme Michaux-Chevry [...].

(Le Monde, 03/08/96)

Qu'ils spécifient une circonstance de la prédication principale, ou qu'ils expriment une circonstance au niveau de l'énonciation ${ }^{20}$, syntagmes infinitifs et gérondifs assurent un lien avec le contexte antérieur tout en signalant une progression à l'intérieur du parcours énonciatif annoncé au préalable ${ }^{21}$. C'est en ce sens que nous les traitons comme des structures cadratives susceptibles de retenir la phase médiane du procès énonciatif.

\subsection{Les locutions adverbiales, marqueurs de reconsidération} adverbiales marqueurs de reconsidération (Rossari, 1990; 1993) comme en fin de

19. Ces verbes s'opposent ainsi à voir qui est neutre par rapport au critère de l'agentivité. Cf. Willems (I983), Willems et Defrancq (2000).

20. Voir Halmøy (2003: chap. VII) quand le gérondif intervient au niveau de l'énonciation.

21. Concernant le rôle textuel du gérondif, notre analyse est fondée sur les travaux de Kleiber et Theissen (2006), Kleiber (2008). 
compte, au bout du compte, finalement, lesquels peuvent exprimer aussi une relation dynamique de parcours (Lenepveu, 2007b) et signaler la phase finale du procès énonciatif. Soit tout d'abord un exemple, déjà partiellement cité, qui illustre la possibilité pour à première vue d'entrer en contraste avec des marques lexicales d'opposition (mauvais calcul) puis avec un marqueur de reconsidération au bout du compte:

[44] À première vue, on pourrait penser que le réchauffement devrait bénéficier à cet oiseau atypique qui réussit le tour de force de se reproduire durant le terrible hiver de l'Antarctique. En effet, températures plus clémentes riment avec réduction de la banquise et, par conséquent, du long chemin - environ 150 kilomètres - séparant la colonie de l'océan nourricier. Les parents, qui se relaient pour aller se ravitailler et rapporter de quoi manger à leur oisillon unique, ont donc moins d'efforts à fournir. Mauvais calcul, explique Henri Weimerskirch. «On s'est aperçu que la diminution de la glace de mer et l'augmentation de la température de surface de l'océan entrainaient une baisse du nombre de krills, ces petites crevettes qui sont la base de la nourriture tant des manchots que des poissons dont se nourrissent aussi les manchots. En fait, quand la température de la mer s'élève, les empereurs ont probablement plus de mal à trouver de quoi manger.» Au bout du compte, les gains éventuels dans le taux de reproduction que l'on est en droit d'espérer du raccourcissement de la banquise ne contrebalancent pas les pertes en effectifs dues à la disette.

(Le Monde, I2/05/oI)

À première vue introduit un premier jugement présenté comme provisoire. Mauvais calcul, en introduisant la prise en compte d'arguments divergents, signale la phase médiane du procès énonciatif et au bout du compte indique la synthèse de ce qui est pris en compte. Suite au parcours effectué et qui était annoncé avec à première vue, le responsable de la reconsidération est amené, au terme du parcours, à adopter un autre point de vue, lequel se donne cette fois comme stabilisé.

Un second exemple fait apparaître plus nettement le caractère progressif du passage de la phase intermédiaire à la phase finale, exemple qui ne met pas en contraste le syntagme gérondif en regardant bien avec l'adverbe finalement, mais les combine tous deux pour «orienter» vers la borne finale du parcours annoncé avec à première vue:

[45] À première vue, on peine à distinguer ce qui a changé; en plaçant côte à côte l'ancien et le dernier modèle, on pourrait jouer au jeu des sept erreurs. Et encore, il n'est pas dit que l'on parviendrait à déceler autant de divergences.

En regardant bien, on découvre finalement que les phares du coupé ont adopté les optiques «duo-elliptiques» inaugurées l'an passé à bord du cabriolet, et que les feux arrière ont été très légèrement redessinés, comme les ailes avant, la grille du radiateur et le bouclier de protection.

(Le Monde, o8/04/o2) 
Enfin, les locutions résultatives tout compte fait, tout bien considéré, tout bien réfléchi, tout bien pesé... également marqueurs de reconsidération, permettent de retenir la phase résultante du procès énonciatif qui consiste à considérer une situation dans son ensemble et qui parvient cette fois-ci à un jugement totalement stabilisé. Suivant les analyses de Rossari (1993), la valeur résultative de ces locutions s'explique par leur structure syntaxique interne passive et par la présence du quantificateur tout. Borné par le quantificateur tout, le procès exprimé par le verbe est en effet perçu comme un accomplissement et la structure passive de ces locutions, qui suppose un sujet responsable de l'opération intellectuelle exprimée par le verbe (compter, considérer, peser...), marque l'état résultant de cet accomplissement ${ }^{22}$. Soit les exemples:

[46] [...] à regarder de plus près les offres, les consommateurs, qui doivent souvent, en plus des points qu'ils ont accumulés, payer une somme complémentaire pour acquérir leur nouveau téléphone, se sont rendu compte qu'ils pouvaient payer in fine plus cher le même produit qu'un nouveau client. Tout compte fait, ils auraient intérêt à résilier leur contrat et à se présenter dans un magasin comme un client à conquérir. (Le Monde, o9/II/oI)

Notons qu'on observe le même type de mise en corrélation pour les locutions au premier abord et de prime abord, proches sémantiquement de à première vue et susceptibles de retenir aussi la phase initiale du procès énonciatif que nous cherchons à mettre en évidence ${ }^{23}$. Par exemple:

[47] Au premier abord, l'objectif de maintenir l'Europe proche des citoyens semble une évidence. Mais, à y regarder de plus près, on perçoit qu'il s'agit de l'un des défis majeurs de la construction européenne [...].

(Le Monde, $\mathrm{I} 4 / \mathrm{ro} / \mathrm{oz}$ )

[48] De prime abord, ce document n'est guère embarrassant pour le gouvernement. Ce dernier peut arguer qu'il est conscient des inégalités de la société française et que ses réformes fiscales ont précisément pour but de les résorber. À lire le rapport de l'Insee, cependant, on comprend pourtant que ces arguments n'épuisent pas le débat. Quels que soient les effets redistributifs des mesures envisagées par le gouvernement, les inégalités de patrimoine continuent, envers et contre tout, à augmenter, tandis que les inégalités de revenus ont cessé de diminuer.

(Le Monde, 07/10/99)

22. Voir aussi Lenepveu (2006).

23. Ces expressions, bien sûr, ne sont pas substituables dans tous les contextes. On peut également mentionner la locution dans un premier temps comme étant susceptible de fonctionner tantôt comme sériel (Nøjgaard, 1993), tantôt comme «marqueur de phase». Voir, sur cette locution, l'étude de Bras et Schnedecker (à paraitre). Ci-dessous un exemple [i] à titre d'illustration:

[i] Cette division des groupes nationaux en «situations politiques» conduit à conclure dans un premier temps que toutes les combinaisons sont imaginables. Cependant, à y regarder de plus près, on constate que si toutes les combinaisons s'appliquaient [...] (H. Carrère d'Encausse, 1978; citée par Bras et Schnedecker, à paraître). 
Les trois locutions s'opposent ainsi à la locution a priori, laquelle introduit un jugement présenté comme antérieur à toute expérience du locuteur concernant l'état de choses envisagé et sélectionne de ce fait la phase préparatoire du procès, c'est-à-dire la phase antérieure à la prise en considération elle-même. Un exemple où $a$ priori est mis en contraste avec la locution tout bien considéré qui retient, pour sa part, la phase résultante de la prise en considération:

[49] Un chercheur isolé est devenu minoritaire, la plupart des travaux sont cosignés. A priori, cela pourrait poser un problème au jury. Il bénéficie cependant avec les travaux multiples qui lui sont remis, d'une vision sur l'auteur qui se retrouve, seul, à l'intersection des différents textes qu'il propose, et donc des différentes préoccupations qu'il manifeste.

Cette difficulté d'appréciation doit être acceptée, car elle tient à la recherche actuelle. Elle manifeste l'intensité des échanges, autrement dit les richesses d'autant plus créées qu'elles sont partagées. Tout bien considéré, elle ne handicape pas vraiment le choix. (Le Monde, 29/o5/or)

Chacune des phases du procès est susceptible d'être sélectionnée au moyen d'une classe d'expressions qui jouent un rôle particulier dans l'organisation du texte, ce que nous représentons au moyen de la figure 2 ci-dessous. Des modulations contextuelles restent bien sûr possibles pour certaines de ces expressions. Si on admet ainsi que l'expression réflexion faite, de par sa structure passive, ne peut exprimer que la phase résultante du procès énonciatif, à la réflexion peut contribuer à exprimer, en fonction d'éléments contextuels, tantôt la phase médiane ( «à la réflexion, on peut se demander si...»), tantôt la phase finale («à la réflexion, on s'est dit que...»).

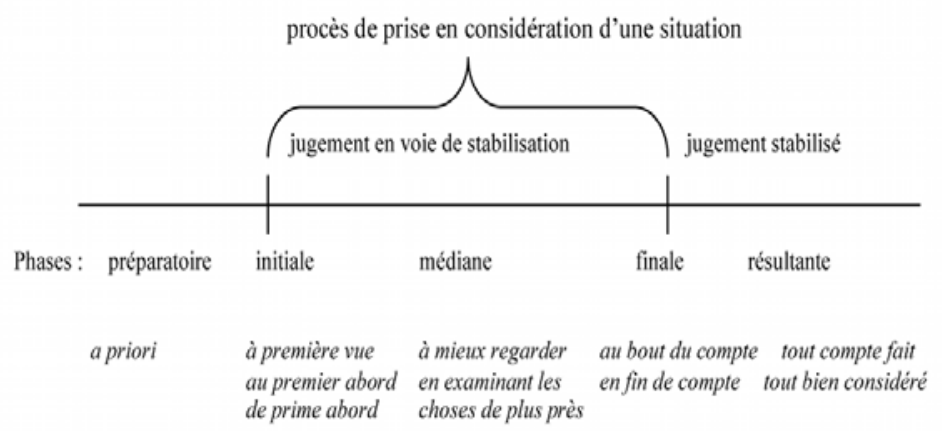

Fig. 2: locutions permettant de sélectionner les phases du procès énonciatif de prise en considération d'une situation

\section{Conclusion}

Nous avons montré comment, à partir de la locution à la première vue et des constructions similaires ( 
premiere veue»...), s'était constituée la locution à première vue dans son emploi endophrastique extra- ou intra-prédicatif, puis dans son emploi exophrastique extra-prédicatif de marqueur de point de vue. Dans cet emploi dit «subjectif», $\grave{a}$ première vue marque, du point de vue énonciatif, le caractère provisoire du jugement énoncé, jugement non pas «emprunté» à autrui, mais ramené à «une première impression », d'où un effet d'atténuation et de distanciation, et la cooccurrence fréquente de la locution avec le conditionnel et/ou certains verbes d'attitude propositionnelle.

En présentant ainsi le point de vue qu'elle introduit comme provisoire et en attente de confirmation, la locution anticipe sur la suite du texte et peut entrer en corrélation avec des expressions qui expriment une évolution du jugement. On a pu ainsi montrer que la locution s'inscrit dans une structure aspectuelle de dicto qui est celle d'un procès énonciatif de prise en considération d'une situation en vue de constituer un jugement stabilisé, chacune des phases de ce procès étant susceptible d'être sélectionnée au moyen d'une classe d'expressions qui jouent un rôle spécifique dans la structuration du texte.

Sur le plan de la structuration textuelle, la locution à première vue présente un fonctionnement que l'on peut dire «doublement cadratif» au sens où elle ouvre un cadre correspondant à la phase initiale du procès énonciatif de prise en considération d'une situation et où, simultanément, elle ouvre le cadre englobant du procès énonciatif lui-même, qui conduit le lecteur à attendre d'autres phases correspondant à une reconsidération de ce point de vue initial donné comme provisoire. À ce titre, elle fonctionne comme un marqueur d'anticipation textuelle.

\section{Bibliographie:}

AвoudA, L. 20or. Les emplois journalistique, polémique, et atténuatif du conditionnel. Un traitement unitaire. In P. Dendale et L. TASMOWSKI (eds), Le conditionnel en français. Recherches linguistiques 25. Metz: Université Paul Verlaine: 277-294.

Anscombre, J.-C. et al. 2009. Apparences, indices et attitude énonciative: le cas de apparemment. Langue française I6I : 39-58.

Aristote, édition 1967. Rhétorique I. Trad. M. Dufour. Paris: Les Belles Lettres.

Birkelund, M., Nølke, H. et Therkelsen, R. (eds) 2009. Langue française: La polyphonie linguistique 164 . Paris: Larousse.

Borillo, A. 2004. Les «adverbes d'opinion forte» selon moi, à mes yeux, à mon avis... : point de vue subjectif et effet d'atténuation. Langue française $\mathrm{I}_{42}: 3 \mathrm{I}^{-} 4 \mathrm{O}$.

Bourdin, P. 1988. Sembler et paraître, ou les deux visages de l'apparence. Semantikos ıo $(\mathrm{I}-2): 45^{-67}$.

Bras, M. et Schnedecker, C. à paraître. Dans un (premier + second $\left.+n^{\text {ième }}\right)$ temps et les relations de discours: de l'élaboration à la contre-argumentation. Linguistic and Psycholinguistic approaches to Text Structuring (LPTSog). Paris : 2I-23 septembre 2009. 
Chafe, W. 1986. Evidentiality in English Conversation and in Academic Writing. In W. Chafe et J. Nichols (eds), Evidentiality: The Linguistic Coding of Epistemology. Norwood: Ablex Publishing Company: 26I-272.

Charolles, M. I997. L'encadrement du discours: univers, champs, domaines et espaces. Cabier de Recherche Linguistique 6. LANDISCO, URA-CNRS I035, Université de Nancy 2: I-73.

Combettes, B. 1995. Approche diachronique des adverbiaux contextuels. $\operatorname{LINX}_{32}: 33^{-5}$.

Combettes, B. et Kuyumcuyan, A. 2007. La formation des modalisateurs en français : le cas des locutions formées sur vérité. Langue française 156: 76-92.

Dendale, P. et Tasmowski, L. 1994. Présentation: l'évidentialité ou le marquage des sources du savoir. Langue française $102:$ 3-7.

Ducrot, O. 1984. Le dire et le dit. Paris : Éditions de Minuit.

Ducrot, O. 1995. Les modificateurs déréalisants. Journal of Pragmatics 24: 145-165.

Fuchs, C. 1989. Introduction: la polysémie de pouvoir. Langue française 84: 4-8.

Gosselin, L. 20oI. Relations temporelles et modales dans le conditionnel journalistique. In P. Dendale et L. TAsmowski (eds), Le conditionnel en français. Recherches linguistiques 25. Metz: Université Paul Verlaine : 45-66.

Gosselin, L. 20Io. Les relations entre périphrases aspectuelles et conjugaisons en français. In N. Flaux, D. Stosic et C. Vet (eds), Interpréter les temps verbaux. Berne: Peter Lang: 33-6I.

Gosselin, L. 20II. L'aspect de phase en français: le rôle des périphrases verbales. Journal of French Language Studies 2I (3).

Guentchéva, Z. (ed.) 1996. L'énonciation médiatisée. Louvain - Paris: Peeters.

Guimier, C. 1996. Les adverbes du français, le cas des adverbes en -ment. Paris - Gap: Ophrys.

HaIllet, P.-P. 2002. Le conditionnel en français: une approche polyphonique. Paris - Gap: Ophrys.

Halmøy, O. 2003. Le gérondif en français. Paris - Gap: Ophrys.

ImBs, P. puis Quemada, B. (dir.) I97I-I994. Trésor de la langue française: dictionnaire de la langue $d u X I X^{\mathrm{e}}$ et $d u X X^{\mathrm{e}}$ siècle. Paris: Éditions du CNRS puis Gallimard.

KLeIBER, G. 2008. Le gérondif: de la phrase au texte. In O. Bertrand et al. (eds), Discours, diachronie, stylistique du français. Études en hommage à Bernard Combettes. Berne : Peter Lang: 107-I23.

Kleiber, G. et Theissen, A. 2006. Le gérondif comme marqueur de cohésion et de cohérence. In F. Calas (ed.), Cohérence et discours. Paris: Presses de l'Université Paris-Sorbonne: 173 -I84.

Korzen, H. et Nølke, H. 1990. Projet pour une théorie des emplois du conditionnel. In Actes $d u$ onzième congrès des romanistes scandinaves. Trondheim, I3-17 août 1990. Université de Trondheim: Institut d'études romanes : 273-300.

Korzen, H. et Nølke, H. 2oor. Le conditionnel: niveaux de modalisation. In P. Dendale et L. TASMOWski (eds), Le conditionnel en français. Recherches linguistiques 25. Metz: Université Paul Verlaine: I25-146. 
Kronning, H. 2004. Modalité et médiation épistémiques. In R. Delamotte-Legrand (ed.), Les médiations langagières. Des faits de langue aux discours. Mont-Saint-Aignan: Publications de l'Université de Rouen : 35-66.

LAZARD, G. 1956. Caractères distinctifs de la langue tadjik. Bulletin de la Société de linguistique de Paris 52 (I) : II7-I86.

LENEPVEU, V. 2006. Structure grammaticale et valeur sémantico-pragmatique des locutions adverbiales de reformulation tout compte fait, tout bien considéré, tout bien pesé... In J. François et S. Mejri (eds), Composition syntaxique et figement lexical. Bibliothèque de Syntaxe \& Sémantique 3. Caen: Presses universitaires de Caen: I87-202.

LENEPVEU, V. 2007a. Intensification et opposition: l'adjectif intensif à valeur argumentative. Travaux de linguistique 55: 45-60.

LENEPVEu, V. 2007b. De la quantification à l'argumentation: le substantif compte dans les locutions adverbiales. Verbum XXIX (3-4): 305-317.

MARTIN, R. 1987. Langage et croyance: les «univers de croyance» dans la théorie sémantique. Bruxelles: Mardaga.

Molinier, C. 2009. Les adverbes d'énonciation. Comment les définir et les sous-classifier? Langue française I6I : 9-22.

Molinier, C. et LeVrier, F. 2000. Grammaire des adverbes, description des formes en -ment. Genève - Paris : Droz.

Myong Soon, K. 2004. Une description des marqueurs évidentiels on dit que et on dirait que. Travaux de linguistique 48: 41-52.

NøjGAARD, M. 1992-1995. Les adverbes français. Essai de description fonctionnelle. Historiskfilosofiske Meddelelser 66. Copenhague: Munksgaard. 3 vol.

NøLKE, H. 1983. Les adverbes paradigmatisants: fonction et analyse. Revue Romane, numéro spécial 23. Copenhague: Akademisk Forlag.

NøLke, H. 1994a. Linguistique modulaire: de la forme au sens. Louvain - Paris: Peeters.

NøLKE, H. 1994b. La dilution linguistique des responsabilités. Essai de description polyphonique des marqueurs évidentiels $i l$ semble que et il paraît que. Langue française $102: 84-94$.

Nølke, H., Fløttum, K. et NorÉn, C. 2004. Scapoline. La théorie scandinave de la polyphonie linguistique. Paris: Kimé.

Rossari, C. 1990. Projet pour une typologie des opérations de reformulation. Cabiers de linguistique française II : 345-359.

Rossari, C. 1993. À propos de l'influence de la composition morphologique d'une locution sur son fonctionnement sémantico-pragmatique. Cabiers de linguistique française 14 : I5O-I7I.

Schnedecker, C. 2002. Premier, principal, primordial: des adjectifs qui sortent du rang. Langue française $136: 89-\mathrm{IO} 3$.

TOURnadre, N. 2004. Typologie des aspects verbaux et intégration à une théorie du TAM. Bulletin de la Société de linguistique de Paris XCIX (I) : 7-68.

TraugotT, E.C. I995. Subjectification in grammaticalisation. In D. STEIN et S. WRIGHT (eds), Subjectivity and subjectivisation. Cambridge: Cambridge University Press: $3 \mathrm{I}^{-54}$. 
Vet, C. 1988. Compte rendu critique de Wallace Chafe \& Johanna Nichols (eds) (1986). Canadian Journal of Linguistics 33 (I) : 65-67.

VET, C. 2002. Les adverbes de temps: composition lexicale et «coercion». In B. LACA (ed.), Temps et aspect. De la morphologie à l'interprétation. Paris: Presses universitaires de Vincennes: 179-192.

VET, C. 2008. Six traits sémantiques suffisent à décrire tous les temps du français. In M. BIRKelund, M.-B. Mosegaard Hansen et C. Norén (eds), L'énonciation dans tous ses états. Mélanges offerts à Henning Nølke. Berne: Peter Lang: 45-47I.

Willems, D. 1983. Regarde voir: les verbes de perception visuelle et la complémentation verbale. In E. RoEgiest et L. TASMOwski (eds), Verbe et phrase dans les langues romanes. Mélanges offerts à Louis Mourin. Gent: Romanica Gandesia: $147-158$.

Willems, D. et Defrance, B. 200o. L'attribut de l'objet et les verbes de perception. Langue française $127: 6$-21. 